\title{
Article \\ Multivariate Exploratory Comparative Analysis of LaLiga Teams: Principal Component Analysis
}

\author{
Claudio A. Casal ${ }^{1, *(\mathbb{D}}$, José L. Losada ${ }^{2}\left(\mathbb{D}\right.$, Daniel Barreira ${ }^{3}$ and Rubén Maneiro ${ }^{4, *}$ \\ 1 Department of Science of Physical Activity and Sport, Catholic University of Valencia "San Vicente Mártir", \\ 46900 Valencia, Spain \\ 2 Department of Social Psychology and Quantitative Psychology, University of Barcelona, \\ 08001 Barcelona, Spain; jlosada@ub.edu \\ 3 Centre of Research, Education, Innovation and Intervention in Sport (CIFI2D), Faculty of Sport, \\ University of Porto, 4099-002 Porto, Portugal; dbarreira@fade.up.pt \\ 4 Department of Science of Physical Activity and Sport, Pontifical University of Salamanca, \\ 37001 Salamanca, Spain \\ * Correspondence: ca.casal@ucv.es (C.A.C.); rmaneirodi@upsa.es (R.M.)
}

check for

updates

Citation: Casal, C.A.; Losada, J.L.; Barreira, D.; Maneiro, R. Multivariate Exploratory Comparative Analysis of LaLiga Teams: Principal Component Analysis. Int. J. Environ. Res. Public Health 2021, 18, 3176. https:// doi.org/10.3390/ijerph18063176

Academic Editors: Paul Tchounwou,

Markel Rico-González and

José Pino-Ortega

Received: 7 February 2021

Accepted: 16 March 2021

Published: 19 March 2021

Publisher's Note: MDPI stays neutral with regard to jurisdictional claims in published maps and institutional affiliations.

Copyright: (C) 2021 by the authors Licensee MDPI, Basel, Switzerland. This article is an open access article distributed under the terms and conditions of the Creative Commons Attribution (CC BY) license (https:// creativecommons.org/licenses/by/ $4.0 /)$.

\begin{abstract}
The use of principal component analysis (PCA) provides information about the main characteristics of teams, based on a set of indicators, instead of displaying individualized information for each of these indicators. In this work we have considered reducing an extensive data matrix to improve interpretation, using PCA. Subsequently, with new components and with multiple linear regression, we have carried out a comparative analysis between the best and bottom teams of LaLiga. The sample consisted of the matches corresponding to the 2015/16, 2016/17 and 2017/18 seasons. The results showed that the best teams were characterized and differentiated from bottom teams in the realization of a greater number of successful passes and in the execution of a greater number of dynamic offensive transitions. The bottom teams were characterized by executing more defensive than offensive actions, showing fewer number of goals and a greater ball possession time in the final third of the field. Goals, ball possession time in the final third of the field, number of effective shots and crosses are the main discriminating performance factors of football. This information allows us to increase knowledge about the key performance indicators (KPI) in football.
\end{abstract}

Keywords: performance analysis; elite football; multivariate analysis; principal component analysis; LaLiga

\section{Introduction}

The identification of performance factors, understood as variables that define some aspect of performance and that help achieve sports success [1], is essential to try to identify the most appropriate behavior patterns that can lead to success [2] and enable the increase and prediction of performance [3,4]. The analysis of the matches will identify those variables related to success [5], and the grouping and combination of these success indicators of different nature will allow the construction of football performance profiles $[4,6]$. To obtain both the indicators and the performance profiles, the discriminant analysis of the game between teams of different levels is a very useful tool. However, we are facing a sport of a complex and dynamic nature, which makes the identification of these performance profiles a very difficult task [7] because the success of the game can be associated with multiple factors (physical, technical, tactical, ... ), some of them being unpredictable or uncontrollable, such as arbitration decisions, individual successes or failures of players, match location, type of competition or even chance.

Football research has turned to a multitude of performance indicators [8], and some studies have tried to identify them through the comparative analysis of successful and unsuccessful teams [9-17]. Some of these works show conflicting results. This may be 
caused, among other things, by the type and size of the sample, the study design, the selection of the variables and the characteristics of the sport itself. It may also be because most studies identify the success of the teams based on the match outcome $[9,16,18-23]$. This discrimination criterion can cause erroneous results because in this sport, in some matches, the team with the best statistical data does not end up getting the victory since in football a single winning play style does not exist. Several teams with different play styles can get similar results. Therefore, it will be necessary to classify the teams, instead of the match outcome, by their position at the end of the season.

To study KPI and performance profiles in football, it would be necessary to perform nomothetic analysis instead of an ideographic one, as the latter would identify the behavior patterns of a unique team and not of the game. It is necessary, therefore, to conduct a longitudinal analysis of all the teams and matches corresponding to one or several regular seasons and classify the teams according to their final position and not based on the match outcome. In this way, the KPI will be more reliable because they will be less mediated by the factors indicated above, and the teams that obtained a higher performance (higher score) at the end of the season can be explained by the fact that they maintained a more effective behavior. Nevertheless, there are few previous studies in this line [11,24-28].

Sometimes, to carry out this type of works, especially when indirect observation methodology is used, we find a very extensive data matrix with many related variables. In this case, it would be beneficial to reduce this matrix for a simpler interpretation and eliminate possible redundant information. However, if the reduction is carried out under some subjective criteria, there is a risk of losing relevant information. Therefore, we need some tool that allows us to objectively reduce the dimensions of a data matrix without losing important information. For this, PCA can be an adequate statistical technique since its aims are to simplify, reduce and structure the initial information obtained [29]. Its application to the tactical analysis of football has been demonstrated in various works with satisfactory results. Specifically, Gómez et al. [30] carried out a study with the aim of identifying the independent and interactive effects of the game location and the final result in the statistics related to the football game according to the area of the field in which they occurred in LaLiga, from 2003 to 2004 and 2007 to 2008 seasons. They identified different profiles in the teams related to the match venue and the match outcome. In the work of Moura et al. [31] two main components were identified in the 2006 World Cup and showed that shots, shots on goal and percentage ball possession are some variables that discriminate among winning, drawing and losing teams. Winter and Pfeiffer [23] identified four dimensions in the UEFA Euro 2012 (game speed, transition play after ball recovery, transition play after ball loss and offense efficiency), concluding that the transition play after losing the ball and the offense efficiency seem to be factors connected directly with the match outcome, as those were important values for a successful discrimination. In [32], the specific aim of their paper was to investigate which factors were most crucial for the match outcome in the Serie A, concluding that shot on target is the performance indicator of the game. In the work of Ric et al. [33], a comparative study of the spatial individual and collective organization of the players was carried out between the first and second half of the game. In the work of Fernández-Crehuet et al. [34] an index was built to measure the performance of Spanish Football league teams, during the 2016/2017 season, combining five dimensions: economic, fans-related, historical, team quality and the season's data. Authors in [35] managed to identify and differentiate various styles of play of the different teams of the Chenesse Soccer Super League during the 2006 season. One style of play denominated possession, other denominated set pieces attack, counterattacking play and, finally, transitional play.

Therefore, we have not found previous works that the PCA have applied to tactically analyze LaLiga teams, during several seasons, and that have determined the level of performance based on the position they occupied in the leaderboard at the end of the season. Nor have they identified and used components to develop a performance model of the teams of different levels. Consequently, we decided to carry out this study to pursue 
the following aims: the first aim of the present study was to reduce the size of a large database and group it into new categories without losing information, through the PCA. The second aim was to perform a comparative and predictive performance analysis among the best and bottom teams of LaLiga, using the KPI of each group.

\section{Materials and Methods}

\subsection{Sample}

In order to carry out this study, 1415 records corresponding to the 2015/16, 2016/17 and 2017/18 seasons of LaLiga have been analyzed. These records belong to the best and bottom teams, ranked according to their final score at the end of the regular league (best teams: the best six teams, qualified in the UEFA Champions League and the Europa League; bottom teams: the three lower teams that descended from a category and the next three worst ranked). Data were obtained from the analysis platform InstatScout and analyzed post event. Instatscout (www.instatscout.com) (accessed on 1 April 2019) is a private platform dedicated to assessing the performance of teams in different world leagues. The information cannot be considered either personal or intimate, as the research consisted solely of naturalistic observations in public places, and it was not anticipated that the recordings would be used in a manner that could cause personal harm. According to the Belmont Report [36], the use of public images for research purpose does not require informed consent or the approval of an ethical committee.

\subsection{Performance Indicators}

To carry out the study, 57 performance indicators were used and divided into three groups, according to the available literature [16,19,28,37-39]: (1) 5 indicators related to goal scoring, (2) 34 related with the offensive phase, and (3) 18 related to the defensive phase (Table 1). The operative definitions can be consulted in www.instatscout.com (accessed on 19 March 2021).

\subsection{Data Reliability}

To ensure the reliability of the data, five randomly selected matches were coded by the authors of this study and then compared with those provided by InStat. The Kappa (K) values obtained ranged from 0.92 to 0.97 .

\subsection{Procedure and Statistical Analysis}

To analyze the game of both groups, a descriptive, comparative and predictive analysis of the performance of the variable "EFFECTIVENESS" was carried out and defined as

$$
\frac{\text { Goals Scored }+ \text { Shots on target }}{\text { Shots }}
$$

The analysis started checking whether the set of the 57 used indicators correctly differentiated the best and bottom teams. For this, a linear discriminant analysis (LDA) was applied, which is a well-established machine learning technique for categories. Its main advantages are that the model is interpretable and that the prediction is simple.

Secondly, in each group of teams, a Principal Component Analysis (PCA) was carried out to reduce the set of indicators and work with a more manageable size, reducing the multicollinearity problem. This technique allows to transform the original information into a new set of variables, called PC, without losing any information. The first transformed PC captures the greatest amount of information, and each subsequent PC explains a reduced amount of information. For the calculation of the main components the variables were standardized (mean 0 and standard deviation 1). When calculated on standardized variables, the main components are eigenvectors that are taken from the correlation matrix, and as many different main components as available variables can be obtained. The number of significant principal components (PC) was determined by the conventional criteria, but only those that cumulatively accounted for $\geq 70 \%$ of the explained variance 
were selected [29]. An additional benefit to PCA is that each PC is uncorrelated, so each one captures distinct information within each individual's data set.

Table 1. Selected performance indicators.

\begin{tabular}{|c|c|c|}
\hline Outcome of Attack & Offence & Defence \\
\hline $\begin{array}{ll}\text { 1. Goals scored, } \\
\text { 2. Shots, } \\
\text { 3. Shots on target, } \\
\text { 4. Effectiveness shots goals, } \\
\text { 5. Effectiveness shots goals and shots } \\
\text { on target. }\end{array}$ & $\begin{array}{l}\text { 1. Attacks, } \\
\text { 2. Positional attacks, } \\
\text { 3. Attacks with shots-positional, } \\
\text { 4. Counterattacks, } \\
\text { 5. Counterattacks with shots, } \\
\text { 6. Set pieces attack, } \\
\text { 7. Attacks with shot set pieces attacks, } \\
\text { 8. Ball possession, } \\
\text { 9. Ball possession in own half, } \\
\text { 10. Ball possession in opponent half, } \\
\text { 11. Ball possession time in the final } \\
\text { 12. Attacking challenges, } \\
\text { 13. Attacking challenges won, } \\
\text { 14. Corners, } \\
\text { 15. Crosses, } \\
\text { 16. Crosses accurate, } \\
\text { 17. Dribbles, } \\
\text { 18. Dribbles successful, } \\
\text { 19. Fouls opponent, } \\
\text { 20. Lost balls, } \\
\text { 21. Lost balls in own half, } \\
\text { 22. Offsides, } \\
\text { 23. Passes, } \\
\text { 24. Passes accurate, } \\
\text { 25. Extra attacking and key passes, } \\
\text { 26. Extra attacking and key passes } \\
\text { 27. Passes forward, } \\
\text { 28. Passes forward accurate, } \\
\text { 29. Passes back, } \\
\text { 30. Passes back accurate, } \\
\text { 31. Passes to the left, } \\
\text { 32. Passes to the left accurate, } \\
\text { 33. Passes to the right, } \\
\text { 34. Passes to the right accurate. } \\
\text { the field, }\end{array}$ & $\begin{array}{l}\text { 1. Challenges, } \\
\text { 2. Challenges won, } \\
\text { 3. Air challenges, } \\
\text { 4. Air challenges won, } \\
\text { 5. Defensive challenges, } \\
\text { 6. Defensive challenges won, } \\
\text { 7. Fouls, } \\
\text { 8. Goals conceded, } \\
\text { 9. Interceptions, } \\
\text { 10. Interceptions in opposition half, } \\
\text { 11. Picking up free balls, } \\
\text { 12. Picking up free balls in opposition } \\
\text { 13. Recovered balls, } \\
\text { 14. Recovered balls in opposition half, } \\
\text { 15. Tackles, } \\
\text { 16. Tackles successful, } \\
\text { 17. Red cards, } \\
\text { 18. Yellow cards. }\end{array}$ \\
\hline
\end{tabular}

Following this, the orthogonal varimax rotation (Varimax method) performance by Kaiser [40] was performed to determine the contribution of the original categories to the variance explained for each extracted $P C$ as well as improving interpretability. The original categories that demonstrated $\mathrm{PC}$ loadings $\left(\mathrm{PC}_{\mathrm{L}}\right.$; i.e., eigenvectors of the covariance matrix) that exceeded \pm 0.70 were considered indicative of a well-defined relationship with the extracted PC [41-43]. The loadings can be interpreted as the weight/importance of each variable in each component; therefore, they help to know what type of information each of the components collects. The purpose of the calculation of the loadings is to identify the linear combinations that best represent the variables $X_{1}, \ldots, X_{p}$. Sean $\left(Z_{1}, Z_{2}, \ldots\right.$, $Z_{M}$ ), where $M<p$ is linear combinations of the original $\mathrm{p}$ variables, that is $Z m=\sum j=$ $1 p \phi j m X j$, where $\phi 1 m, \phi 2 m, \ldots, \phi p m, \phi 1 m, \phi 2 m, \ldots, \phi p m$ are the constants, or loadings, of the main components (for example, $\phi_{11}$ would correspond to the first loading of the first main component).

To finish, an analysis of variance (ANOVA) was carried out to check the differences between the different main components. By means of multiple linear regression, a pre- 
diction model was also constructed for each group, formed by the explained variable "EFFECTIVENESS", and the main components found in each group.

$$
\begin{gathered}
Y_{t}=\beta_{0}+\beta_{1} X_{1}+\beta_{2} X_{2}+\ldots+\beta_{p} X_{p}+\varepsilon+ \\
Y_{t} \text { explained variable; } X_{p} \text { principals componts of each variable }
\end{gathered}
$$

This model will allow us to identify the set of variables that have greater influence on the performance of each group and check if there are differences between the styles of play between both groups of teams.

To carry out the statistical analysis, the R program (v.3.4.1) was used, using the MASS [44], stats and citatiom ("factomineR and factoextra") libraries.

\section{Results}

The results obtained from the linear discriminant analysis (LDA) were $85.63 \%$ well classified and $14.36 \%$ poorly classified, which reveals that the indicators used correctly classified the teams as best and bottom.

\subsection{Best Teams PCA Results}

Figure 1 shows the screeplot, the eigenvectors and the accumulation of the variance explained by each PC. The eight eigenvectors produced by the PCA explained $70.1 \%$ of the total variance, and its eigenvalues were higher than 1 (see Table 2).

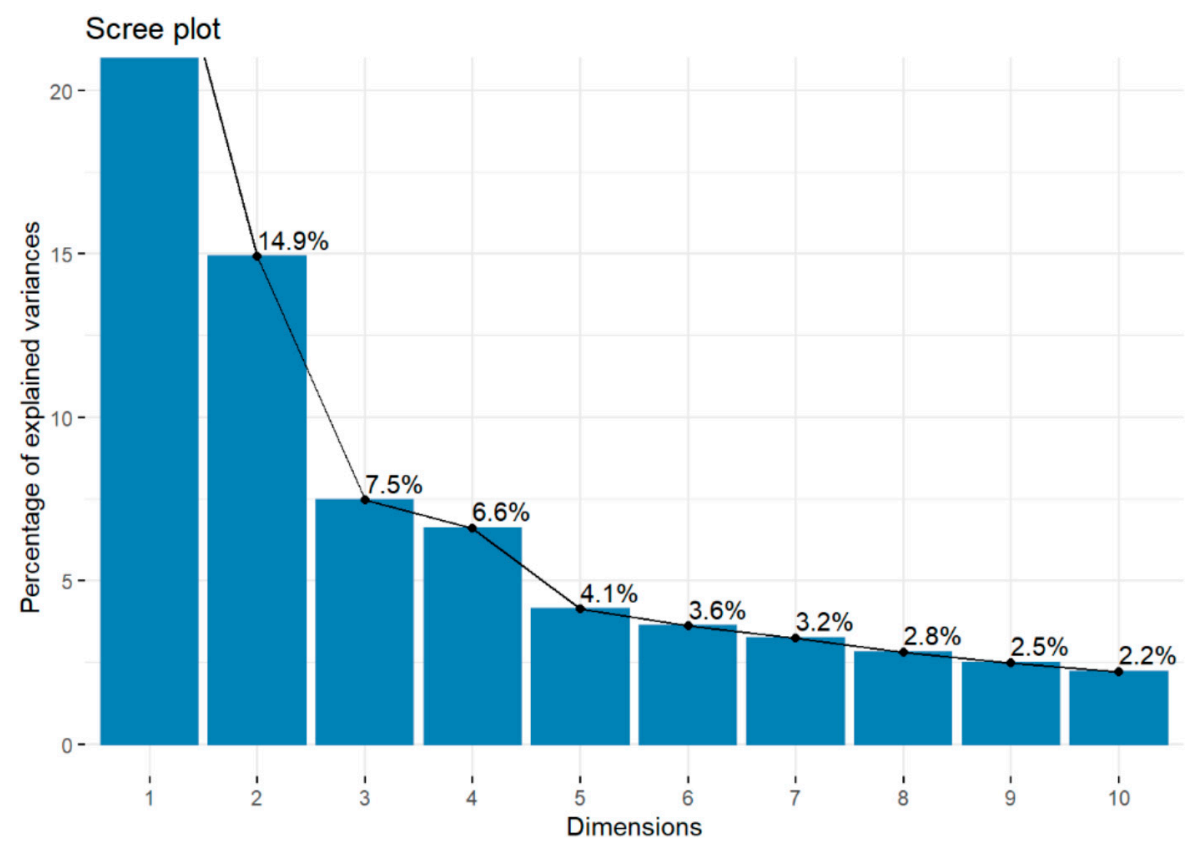

Figure 1. Screeplot of best teams.

Table 2. Components and total variance explained.

\begin{tabular}{ccccccccc}
\hline Parameters & PC1 & PC2 & PC3 & PC4 & PC5 & PC6 & PC7 & PC8 \\
\hline Standard deviation & 3.94 & 2.91 & 2.06 & 1.93 & 1.53 & 1.43 & 1.35 & 1.26 \\
Proportion of Variance & 0.27 & 0.14 & 0.07 & 0.06 & 0.04 & 0.03 & 0.03 & 0.02 \\
Cumulative Proportion & 0.27 & 0.42 & 0.49 & 0.56 & 0.60 & 0.64 & 0.67 & 0.70 \\
\hline
\end{tabular}

The component loadings after rotation is illustrated in Table 3. Applying the Varimax rotation method maximizes the variance of the matrix of charges so that the values are more interpretable. The "rotated" matrix provides the loadings of the main components, and each column contains the vector of loadings for each main component. 
Table 3. PCA factorial matrix of best teams.

\begin{tabular}{|c|c|c|c|c|c|c|c|c|}
\hline Categories & PC1 & PC2 & PC3 & PC4 & PC5 & PC6 & PC7 & PC8 \\
\hline GOALS SCORED & -0.08 & 0.09 & -0.26 & 0.23 & 0.08 & -0.14 & 0.10 & -0.09 \\
\hline GOALS CONCEDED & 0.02 & -0.06 & 0.10 & 0.02 & -0.14 & 0.01 & 0.01 & 0.16 \\
\hline YELOW CARD & 0.07 & -0.01 & 0.09 & -0.06 & -0.07 & 0.09 & 0.00 & -0.01 \\
\hline RED CARD & 0.05 & 0.03 & 0.05 & -0.01 & -0.14 & 0.04 & -0.08 & 0.11 \\
\hline CORNERS & 0.10 & -0.09 & 0.20 & 0.19 & 0.07 & 0.11 & -0.02 & -0.15 \\
\hline SHOTS SET PIECES ATTACK & -0.10 & -0.07 & 0.13 & 0.19 & -0.07 & -0.11 & 0.02 & 0.15 \\
\hline OFFSIDES & -0.02 & -0.02 & -0.06 & 0.11 & -0.03 & -0.12 & 0.14 & 0.00 \\
\hline FOULS & 0.10 & -0.00 & 0.09 & 0.00 & 0.04 & 0.12 & 0.05 & 0.00 \\
\hline FOULS OPPONENT & -0.02 & -0.09 & -0.01 & -0.05 & -0.31 & 0.07 & 0.23 & -0.01 \\
\hline POSSESSION & -0.22 & -0.06 & -0.03 & -0.10 & 0.01 & -0.01 & -0.03 & -0.01 \\
\hline POSSESSION OWN HALF & -0.09 & 0.02 & -0.21 & -0.14 & -0.03 & -0.19 & -0.05 & 0.43 \\
\hline POSSESSION OPPONENT HALF & -0.18 & 0.03 & 0.13 & -0.05 & 0.05 & 0.12 & 0.08 & 0.05 \\
\hline POSSESSION FINAL THIRD FIELD & -0.15 & -0.04 & 0.22 & 0.06 & 0.05 & 0.15 & 0.05 & -0.32 \\
\hline SHOTS ON TARGET & -0.13 & 0.04 & -0.12 & 0.31 & 0.04 & -0.07 & 0.06 & -0.04 \\
\hline EFF. SHOTS GOALS & -0.01 & -0.09 & -0.30 & 0.11 & 0.09 & -0.18 & 0.15 & -0.11 \\
\hline EFFECTIVENESS & -0.01 & 0.09 & -0.30 & 0.14 & 0.08 & -0.15 & 0.14 & -0.13 \\
\hline ATTACKS & -0.10 & -0.25 & 0.08 & 0.04 & -0.03 & -0.15 & -0.02 & 0.05 \\
\hline POSITIONAL ATTACK & -0.13 & -0.19 & 0.06 & -0.11 & -0.03 & -0.15 & 0.09 & -0.00 \\
\hline SHOTS PIECES ATTACK & -0.16 & 0.00 & 0.04 & 0.16 & 0.02 & -0.01 & 0.06 & -0.01 \\
\hline COUNTERATTACKS & 0.06 & -0.11 & -0.07 & 0.21 & 0.10 & 0.03 & -0.33 & 0.00 \\
\hline SHOTS COUNTERATTACK & -0.03 & 0.03 & -0.03 & 0.29 & 0.13 & 0.06 & -0.23 & -0.06 \\
\hline SET PIECES ATTACK & -0.07 & -0.15 & 0.20 & 0.18 & -0.13 & -0.11 & 0.07 & 0.17 \\
\hline PASSES & -0.23 & -0.06 & -0.06 & -0.13 & 0.05 & 0.01 & -0.04 & -0.06 \\
\hline PASSES ACCURATE & -0.23 & -0.02 & -0.06 & -0.11 & 0.04 & 0.04 & -0.02 & -0.06 \\
\hline EXTRA AT. KEY PASSES & -0.15 & -0.01 & -0.11 & 0.20 & -0.05 & -0.07 & 0.02 & 0.06 \\
\hline EXTRA AT. KEY PASSES ACCURATE & -0.15 & -0.01 & -0.11 & 0.20 & -0.05 & -0.07 & 0.02 & 0.06 \\
\hline CROSSES & -0.10 & -0.11 & 0.27 & 0.06 & 0.12 & -0.06 & -0.00 & -0.01 \\
\hline CROSSES ACCURATE & -0.08 & -0.08 & 0.22 & 0.11 & 0.13 & -0.08 & -0.02 & -0.04 \\
\hline CHALLENGES & 0.10 & -0.28 & -0.08 & -0.00 & 0.00 & 0.08 & 0.07 & -0.02 \\
\hline CHALLENGES WON & 0.05 & -0.29 & -0.12 & 0.03 & -0.02 & 0.11 & 0.16 & -0.06 \\
\hline DEFFENCE CHALLENGES & 0.13 & -0.20 & -0.07 & -0.01 & 0.17 & 0.10 & 0.15 & -0.00 \\
\hline DEFFENCE CHALLENGES WON & 0.09 & -0.20 & -0.06 & -0.02 & 0.23 & 0.05 & 0.28 & -0.00 \\
\hline AT.CHALLENGES & 0.04 & -0.28 & -0.07 & 0.00 & -0.18 & 0.04 & -0.03 & -0.03 \\
\hline AIR CHALLENGES & 0.11 & -0.22 & 0.03 & -0.07 & 0.03 & -0.24 & 0.08 & -0.15 \\
\hline AIR CHALLENGES WON & 0.08 & -0.23 & 0.02 & -0.05 & 0.03 & -0.23 & 0.14 & -0.15 \\
\hline DRIBBLES & -0.09 & -0.13 & -0.18 & -0.05 & -0.32 & 0.25 & -0.06 & 0.02 \\
\hline DRIBBLES SUCCESSFUL & -0.10 & -0.11 & -0.18 & 0.08 & -0.33 & 0.25 & -0.04 & 0.00 \\
\hline TACKLES & 0.10 & -0.06 & -0.09 & 0.09 & 0.24 & 0.36 & 0.09 & 0.24 \\
\hline TACKLES SUCCESSFUL & 0.07 & -0.09 & -0.10 & 0.07 & 0.31 & 0.31 & 0.20 & 0.22 \\
\hline
\end{tabular}


Table 3. Cont.

\begin{tabular}{|c|c|c|c|c|c|c|c|c|}
\hline Categories & PC1 & PC2 & PC3 & PC4 & PC5 & PC6 & PC7 & PC8 \\
\hline INTERCEPTIONS & 0.14 & -0.07 & -0.09 & -0.05 & 0.03 & -0.05 & -0.31 & -0.10 \\
\hline INTERCEPTIONS OPPOSITION HALF & -0.01 & -0.16 & 0.01 & 0.02 & 0.07 & -0.00 & -0.29 & -0.01 \\
\hline PICKING UP FREE BALLS & 0.01 & -0.18 & 0.05 & -0.06 & 0.13 & -0.12 & 0.08 & 0.13 \\
\hline $\begin{array}{l}\text { PICKING UP FREE BALL OPPOSITION } \\
\text { HALF }\end{array}$ & -0.12 & -0.14 & 0.15 & 0.02 & 0.11 & -0.03 & 0.04 & 0.17 \\
\hline LOST BALLS & 0.07 & -0.21 & -0.10 & -0.05 & 0.00 & -0.26 & -0.14 & 0.21 \\
\hline LOST BALLS OWN HALF & 0.10 & -0.04 & -0.21 & -0.09 & -0.09 & -0.06 & -0.21 & -0.09 \\
\hline RECOVERED BALL & 0.04 & -0.18 & -0.09 & 0.03 & 0.17 & 0.01 & -0.30 & -0.01 \\
\hline $\begin{array}{l}\text { RECOVERED BALL OPPOSITION } \\
\text { HALF }\end{array}$ & -0.06 & -0.08 & 0.04 & 0.12 & 0.18 & 0.10 & -0.26 & 0.17 \\
\hline PASSES FORWARD & -0.21 & -0.10 & -0.06 & -0.14 & -0.08 & -0.00 & -0.00 & -0.06 \\
\hline PASSES BACK & -0.19 & 0.07 & -0.04 & -0.12 & 0.05 & 0.02 & 0.08 & 0.24 \\
\hline PASSES LEFT & -0.22 & -0.05 & -0.06 & -0.14 & 0.07 & 0.02 & -0.03 & -0.05 \\
\hline PASSES RIGHT & -0.22 & -0.05 & -0.07 & -0.14 & 0.06 & 0.01 & -0.02 & -0.07 \\
\hline PASSES FORWARD ACCURATE & -0.23 & -0.05 & -0.05 & -0.11 & 0.05 & 0.04 & -0.04 & -0.09 \\
\hline PASSES BACK ACCURATE & -0.19 & 0.08 & -0.05 & -0.13 & 0.05 & 0.02 & 0.08 & 0.23 \\
\hline PASSES LEFT ACCURATE & -0.23 & -0.02 & -0.06 & -0.12 & 0.06 & 0.05 & -0.02 & -0.05 \\
\hline PASSES RIGHT ACCURATE & -0.23 & -0.02 & -0.06 & -0.13 & 0.05 & 0.04 & -0.01 & -0.06 \\
\hline
\end{tabular}

Component loadings $\geq$ median contribution appear in bold. EFF: Effectiveness. SH: Shot. AT: Attack.

The information in this table can be summarized as shown in Figure 2. The dashed horizontal line indicates the average contribution value of the different categories in each component. A category with a value greater than this limit is considered important for that component. The dominant category (or categories) in each component was used to subjectively characterize new variables: (a) Passes; (b) Challenges; (c) Attacks effectiveness; (d) Shots; (e) Dribbles; (f) Tackles; (g) Offensive transitions and (h) Possession.

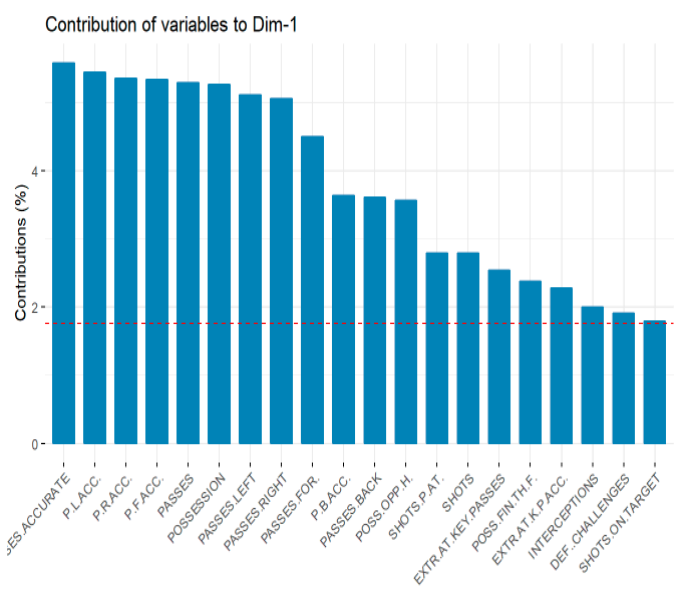

(a). Component 1: Passes

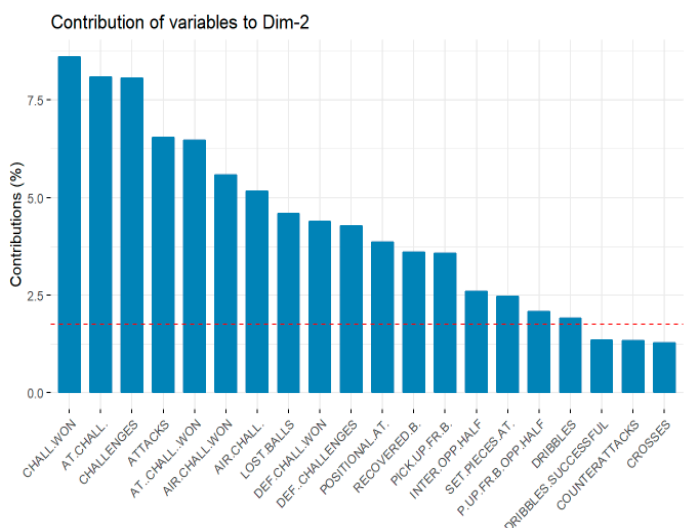

(b). Component 2: Challenges

Figure 2. Cont. 


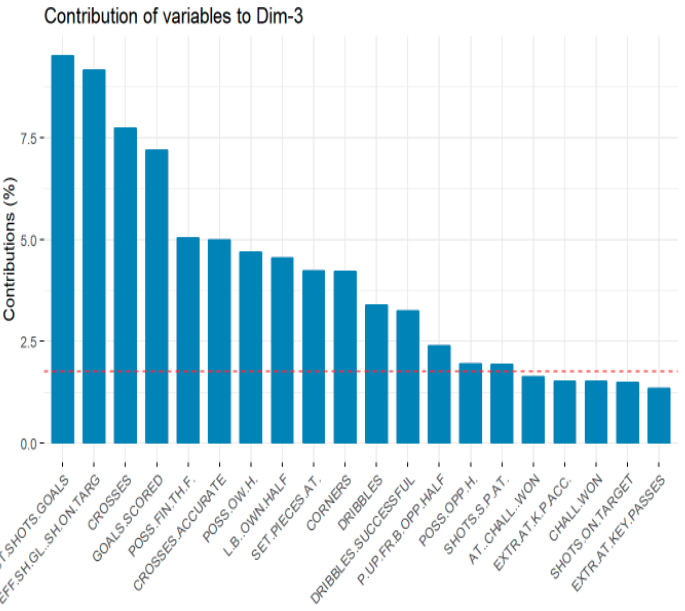

(c). Component 3: Attack effectiveness

Contribution of variables to Dim-5

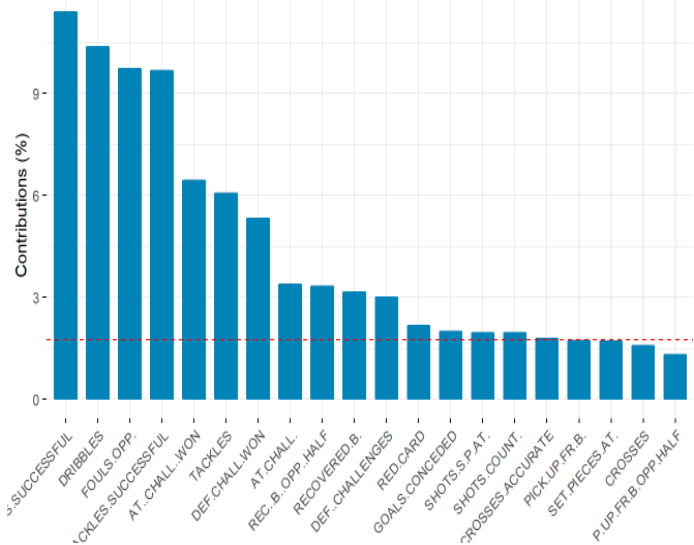

(e). Component 5: Dribbles

Contribution of variables to Dim-7
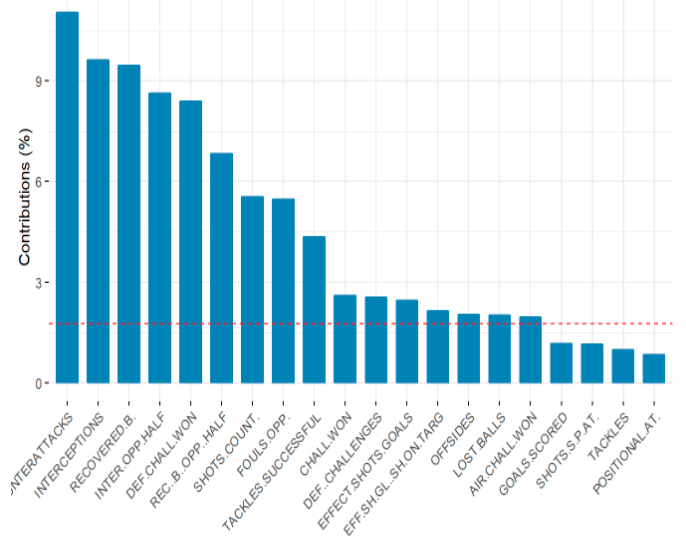

(g). Component 7: Offensive transitions
Contribution of variables to Dim-4

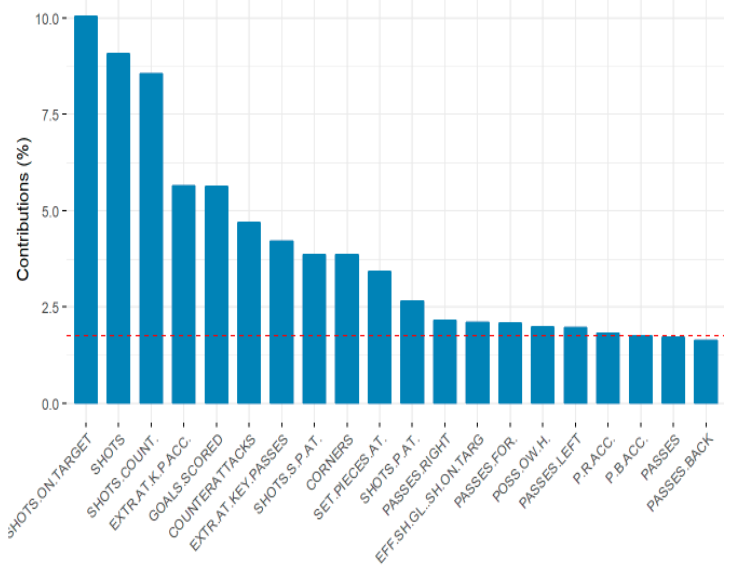

(d). Component 4: Shots

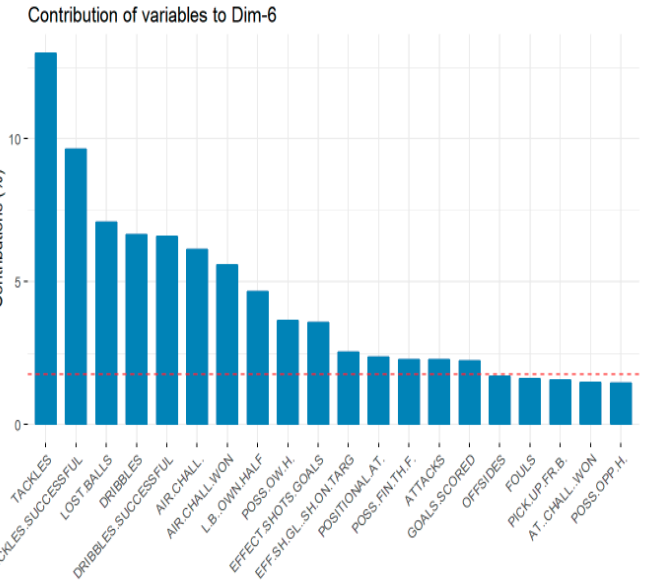

(f). Component 6: Tackles

Contribution of variables to Dim-8

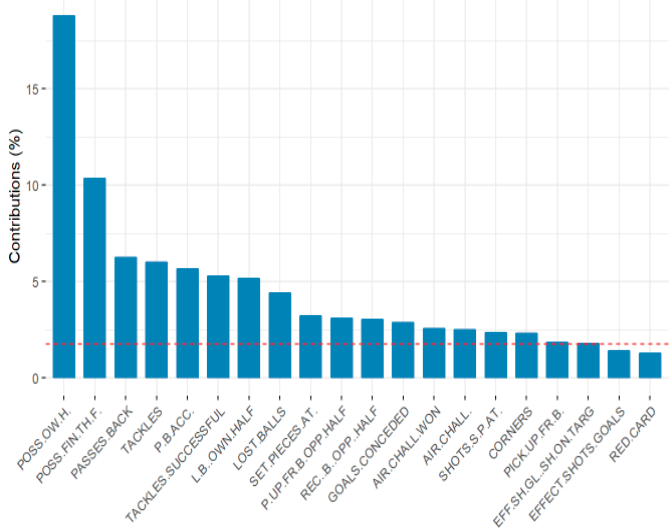

(h). Component 8: Possession

Figure 2. Components and new subjective variables of best teams. (a): Passes; (b): Challenges; (c): Attack effectiveness; (d): Shots; (e): Dribbles; (f): Tackles; (g): Offensive transitions; (h): Possession.

\subsection{Bottom Teams PCA Results}

In bottom teams a screeplot was generated to represent the eigenvectors ordered from highest to lowest (Figure 3). 


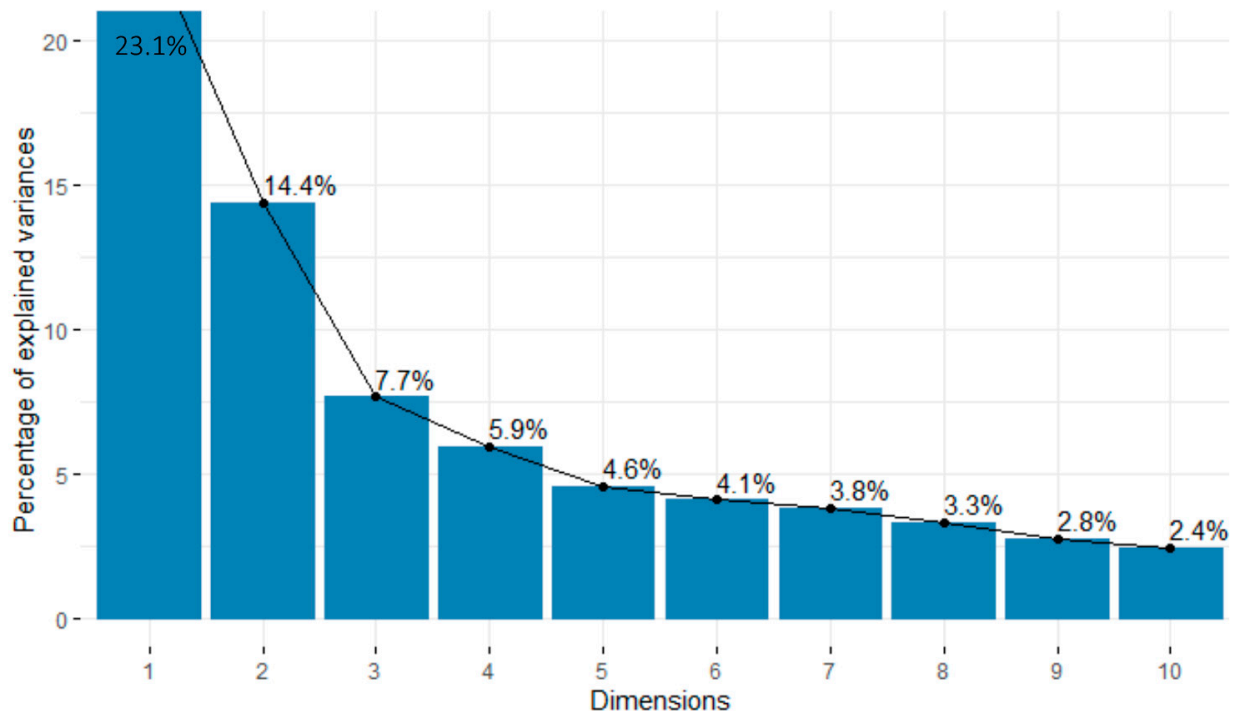

Figure 3. Screeplot for bottom teams.

Taking into account the information of the accumulated variance and that of the eigenvalues, the first nine components that explain $70 \%$ of the variance were used (Table 4). Table 5 shows the bottom teams' component loadings after rotation.

Table 4. Bottom teams' principal components and total variance explained.

\begin{tabular}{cccccccccc}
\hline Parameters & PC1 & PC2 & PC3 & PC4 & PC5 & PC6 & PC7 & PC8 & PC9 \\
\hline Standard deviation & 3.65 & 2.86 & 2.08 & 1.84 & 1.61 & 1.52 & 1.47 & 1.37 & 1.25 \\
Proportion of Variance & 0.23 & 0.14 & 0.07 & 0.05 & 0.04 & 0.04 & 0.03 & 0.03 & 0.03 \\
Cumulative Proportion & 0.23 & 0.37 & 0.45 & 0.51 & 0.56 & 0.60 & 0.63 & 0.67 & 0.70 \\
\hline
\end{tabular}

Table 5. PCA factorial matrix of bottom teams.

\begin{tabular}{lccccccccc}
\hline Categories & PC1 & PC2 & PC3 & PC4 & PC5 & PC6 & PC7 & PC8 & PC9 \\
\hline GOALS SCORED & 0.01 & -0.01 & $\mathbf{0 . 2 1}$ & $-\mathbf{0 . 3 3}$ & -0.05 & $\mathbf{0 . 1 9}$ & $-\mathbf{0 . 1 8}$ & $\mathbf{0 . 1 3}$ & -0.06 \\
\hline GOALS CONCEDED & 0.02 & 0.12 & -0.01 & -0.03 & 0.11 & -0.09 & 0.04 & -0.06 & -0.19 \\
\hline YELOW CARD & 0.03 & -0.06 & 0.02 & 0.03 & 0.04 & -0.00 & -0.07 & 0.00 & 0.12 \\
\hline RED CARD & 0.04 & 0.00 & -0.00 & 0.04 & 0.11 & 0.02 & -0.02 & -0.03 & $-\mathbf{0 . 2 5}$ \\
\hline CORNERS & $\mathbf{- 0 . 1 3}$ & 0.02 & $\mathbf{0 . 1 9}$ & 0.08 & 0.03 & 0.00 & 0.02 & $-\mathbf{0 . 2 5}$ & -0.13 \\
\hline OFFSIDES & -0.01 & -0.05 & -0.06 & -0.09 & -0.09 & 0.12 & 0.06 & 0.08 & 0.04 \\
\hline FOULS & 0.06 & -0.09 & 0.04 & 0.11 & -0.07 & -0.05 & -0.09 & 0.08 & 0.10 \\
\hline FOULS OPPONENT & -0.07 & -0.06 & -0.02 & 0.06 & 0.08 & -0.00 & $-\mathbf{0 . 3 0}$ & 0.15 & 0.04 \\
\hline POSSESSION & $\mathbf{- 0 . 2 4}$ & 0.06 & -0.07 & -0.05 & 0.01 & 0.05 & 0.03 & 0.02 & 0.02 \\
\hline POSSESSION OWN HALF & -0.04 & 0.04 & -0.13 & $\mathbf{- 0 . 1 8}$ & $-\mathbf{0 . 2 5}$ & $-\mathbf{0 . 1 3}$ & $-\mathbf{0 . 2 1}$ & $-\mathbf{0 . 3 7}$ & -0.01 \\
\hline POSSESSION OPPONENT & -0.12 & $\mathbf{0 . 1 4}$ & 0.07 & $\mathbf{0 . 1 4}$ & $-\mathbf{0 . 2 5}$ & -0.12 & $-\mathbf{0 . 1 6}$ & 0.02 & $\mathbf{0 . 2 4}$ \\
HALF & $\mathbf{0 . 1 4}$ & 0.04 & $\mathbf{0 . 1 6}$ & $\mathbf{0 . 2 0}$ & 0.11 & 0.08 & 0.12 & $\mathbf{0 . 3 1}$ & 0.10 \\
\hline $\begin{array}{l}\text { POSSESSION FINAL } \\
\text { THIRD FIELD }\end{array}$ & -0.05 & -0.00 & $\mathbf{0 . 3 0}$ & $\mathbf{- 0 . 2 5}$ & -0.01 & 0.01 & -0.03 & -0.08 & -0.08 \\
\hline SHOTS ON TARGET & 0.06 & -0.02 & 0.10 & $\mathbf{- 0 . 3 1}$ & -0.07 & $\mathbf{0 . 2 5}$ & $-\mathbf{0 . 2 3}$ & $\mathbf{0 . 1 6}$ & -0.01 \\
\hline EFF. SHOTS GOALS & & & & & &
\end{tabular}


Table 5. Cont.

\begin{tabular}{|c|c|c|c|c|c|c|c|c|c|}
\hline Categories & PC1 & PC2 & PC3 & PC4 & PC5 & PC6 & PC7 & PC8 & PC9 \\
\hline EFFECTIVENESS & 0.06 & -0.02 & 0.11 & -0.32 & -0.07 & 0.21 & -0.20 & 0.19 & 0.01 \\
\hline ATTACKS & -0.21 & -0.12 & 0.06 & 0.05 & 0.01 & 0.09 & -0.03 & -0.11 & 0.00 \\
\hline POSITIONAL ATTACK & -0.21 & -0.05 & -0.04 & 0.05 & -0.01 & 0.12 & -0.11 & -0.01 & -0.06 \\
\hline SHOTS PIECES AT. & -0.14 & 0.05 & 0.17 & -0.05 & 0.01 & -0.09 & 0.06 & 0.01 & -0.15 \\
\hline COUNTERATTACKS & -0.00 & -0.15 & 0.11 & -0.11 & 0.00 & -0.04 & 0.24 & -0.11 & 0.23 \\
\hline $\begin{array}{l}\text { SHOTS } \\
\text { COUNTERATTACK }\end{array}$ & 0.00 & -0.03 & 0.19 & -0.17 & -0.01 & -0.08 & 0.23 & -0.02 & 0.07 \\
\hline SET PIECES ATTACK & -0.12 & -0.06 & 0.21 & 0.17 & 0.06 & 0.00 & -0.11 & -0.20 & -0.11 \\
\hline PASSES & -0.24 & 0.07 & -0.13 & -0.07 & 0.02 & 0.08 & 0.07 & 0.04 & 0.00 \\
\hline PASSES ACCURATE & -0.23 & 0.11 & -0.12 & -0.06 & 0.02 & 0.04 & 0.05 & 0.08 & -0.00 \\
\hline EXTRA AT. KEY PASSES & -0.10 & 0.01 & 0.22 & -0.18 & -0.10 & -0.10 & 0.02 & -0.07 & 0.03 \\
\hline $\begin{array}{l}\text { EXTRA AT. KEY PASSES } \\
\text { ACCURATE }\end{array}$ & -0.08 & 0.01 & 0.24 & -0.21 & -0.09 & -0.12 & 0.02 & -0.02 & 0.02 \\
\hline CROSSES & -0.17 & 0.03 & 0.14 & 0.14 & 0.00 & 0.07 & 0.08 & -0.01 & -0.12 \\
\hline CROSSES ACCURATE & -0.13 & 0.01 & 0.15 & 0.12 & -0.00 & 0.05 & -0.00 & 0.03 & -0.20 \\
\hline CHALLENGES & -0.07 & -0.31 & -0.07 & 0.01 & 0.00 & -0.07 & -0.04 & 0.07 & -0.04 \\
\hline CHALLENGES WON & -0.09 & -0.29 & -0.03 & 0.00 & -0.02 & -0.10 & -0.08 & 0.11 & -0.03 \\
\hline $\begin{array}{l}\text { DEFFENCE } \\
\text { CHALLENGES }\end{array}$ & -0.02 & -0.27 & -0.10 & -0.00 & -0.16 & -0.02 & 0.10 & 0.12 & -0.12 \\
\hline $\begin{array}{l}\text { DEFFENCE } \\
\text { CHALLENGES WON }\end{array}$ & -0.04 & -0.25 & -0.06 & 0.04 & -0.27 & -0.00 & 0.03 & 0.13 & -0.10 \\
\hline AT.CHALLENGES & -0.10 & -0.25 & -0.02 & 0.03 & 0.14 & -0.10 & -0.18 & -0.00 & 0.04 \\
\hline AIR CHALLENGES & -0.06 & -0.26 & -0.02 & 0.11 & -0.08 & 0.21 & -0.12 & -0.05 & -0.00 \\
\hline AIR CHALLENGES WON & -0.07 & -0.24 & -0.00 & 0.09 & -0.09 & 0.20 & -0.14 & -0.00 & -0.00 \\
\hline DRIBBLES & -0.10 & -0.09 & -0.05 & -0.13 & 0.28 & -0.33 & -0.11 & 0.03 & 0.10 \\
\hline DRIBBLES SUCCESSFUL & -0.10 & -0.08 & -0.03 & -0.15 & 0.29 & -0.35 & -0.15 & 0.03 & 0.08 \\
\hline TACKLES & 0.05 & -0.11 & -0.07 & -0.11 & -0.18 & -0.31 & 0.21 & 0.16 & -0.21 \\
\hline TACKLES SUCCESSFUL & 0.00 & -0.13 & -0.06 & -0.03 & -0.30 & -0.23 & 0.18 & 0.19 & -0.19 \\
\hline INTERCEPTIONS & 0.07 & -0.15 & -0.05 & -0.09 & 0.07 & 0.12 & 0.10 & -0.18 & 0.33 \\
\hline $\begin{array}{l}\text { INTERCEPTIONS } \\
\text { OPPOSITION HALF }\end{array}$ & -0.07 & -0.12 & 0.07 & 0.02 & 0.02 & 0.05 & 0.09 & -0.15 & 0.25 \\
\hline $\begin{array}{l}\text { PICKING UP FREE } \\
\text { BALLS }\end{array}$ & -0.10 & -0.11 & 0.02 & 0.13 & -0.23 & 0.02 & -0.03 & -0.05 & -0.02 \\
\hline $\begin{array}{l}\text { PICKING UP FREE BALL } \\
\text { OPPOSITION HALF }\end{array}$ & -0.15 & -0.03 & 0.14 & 0.18 & -0.15 & -0.05 & -0.03 & -0.04 & -0.01 \\
\hline LOST BALLS & -0.05 & -0.19 & -0.14 & -0.15 & 0.08 & 0.12 & 0.06 & -0.34 & -0.21 \\
\hline LOST BALLS OWN HALF & 0.03 & -0.09 & 0.20 & -0.23 & 0.05 & 0.06 & 0.04 & -0.28 & -0.24 \\
\hline RECOVERED BALL & -0.08 & -0.18 & 0.01 & -0.08 & -0.07 & 0.05 & 0.24 & -0.04 & 0.28 \\
\hline $\begin{array}{l}\text { RECOVERED BALL } \\
\text { OPPOSITION HALF }\end{array}$ & -0.09 & -0.06 & 0.13 & -0.00 & -0.07 & -0.01 & 0.20 & -0.05 & 0.19 \\
\hline PASSES FORWARD & -0.24 & 0.01 & -0.13 & -0.06 & 0.02 & 0.11 & 0.09 & 0.02 & 0.01 \\
\hline PASSES BACK & -0.11 & 0.18 & -0.08 & -0.03 & -0.28 & -0.16 & -0.20 & -0.12 & 0.11 \\
\hline
\end{tabular}


Table 5. Cont.

\begin{tabular}{lccccccccc}
\hline Categories & PC1 & PC2 & PC3 & PC4 & PC5 & PC6 & PC7 & PC8 & PC9 \\
\hline PASSES LEFT & $\mathbf{- 0 . 2 4}$ & 0.06 & -0.13 & -0.07 & 0.02 & 0.07 & 0.06 & 0.04 & 0.00 \\
\hline PASSES RIGHT & $\mathbf{- 0 . 2 4}$ & 0.07 & -0.13 & -0.06 & 0.01 & 0.08 & 0.07 & 0.04 & 0.00 \\
\hline $\begin{array}{l}\text { PASSES FORWARD } \\
\text { ACCURATE }\end{array}$ & $\mathbf{- 0 . 2 4}$ & 0.08 & -0.11 & -0.06 & 0.04 & 0.05 & 0.06 & 0.07 & -0.00 \\
\hline $\begin{array}{l}\text { PASSES BACK } \\
\text { ACCURATE }\end{array}$ & -0.11 & $\mathbf{0 . 1 8}$ & -0.08 & -0.03 & $\mathbf{- 0 . 2 7}$ & $\mathbf{- 0 . 1 5}$ & $\mathbf{- 0 . 2 0}$ & -0.11 & 0.10 \\
\hline $\begin{array}{l}\text { PASSES LEFT ACCURATE } \\
\text { PASSES RIGHT }\end{array}$ & $\mathbf{- 0 . 2 3}$ & 0.11 & -0.13 & -0.07 & 0.04 & 0.03 & 0.04 & 0.08 & 0.00 \\
ACCURATE & $\mathbf{- 0 . 2 3}$ & 0.11 & -0.11 & 0.07 & 0.01 & 0.04 & 0.05 & 0.07 & -0.01 \\
\hline
\end{tabular}

Component loadings $\geq$ median contribution appear in bold. EFF: Effectiveness. SH: Shot. AT: Attack.

Figure 4 shows the contribution of the different categories in each component and the new subjectively defined category: (a) Passes; (b) Challenges; (c) Shots; (d) Attacks effectiveness; (e) 1 vs 1; (f) Dribbles; (g) Fouls; (h) Possession balls and (i) Offensive transitions.

\subsection{Best Teams ANOVA and Linear Regression Model}

An analysis of variance indicated that there were significant differences in the eight main components (Table 6).

Table 6. ANOVA best teams.

\begin{tabular}{cccccc}
\hline Model & Df Sum & Sq Mean & Sq F & Value & Pr $(>F)$ \\
\hline Pca1 & 8 & 2966 & 370.8 & 183.5 & $<0.000^{* * *}$ \\
Residuals & & 675 & 1364 & 2.0 & \\
\hline
\end{tabular}

Signif. codes: ${ }^{* * *} 0.001$.

It was verified that the residues were distributed randomly around 0 . The ShapiroWilk test was performed to check the normality of the main component residuals. A $p$-value $=0.337$ was obtained. Therefore, they follow normality. For the homoscedasticity of the residues the test of Breusch-Pagan was used with a value of $\mathrm{BP}=1.8871, \mathrm{df}=2$, and a $p$-value $=0.389$, which concludes that there is no evidence of lack of homoscedasticity.

Autocorrelation was studied by means of the DW statistic that showed a result of 1879, with a $p$-value $=0.959$. Therefore, there is no evidence of autocorrelation. The eight main components were entered into a linear regression model (Table 7) to predict the explained variable "EFFECTIVENESS". It can be seen how all the PCs provided different information to the model, with PC3 being the one with the greatest weight $(0.76260)$ and PC1 with the lowest weight (0.03851).

Table 7. Best teams linear regression model.

\begin{tabular}{ccccc}
\hline Components & Estimate Std & Error & $\boldsymbol{t}$ Value & $\operatorname{Pr}(>|\mathbf{t}|)$ \\
\hline (Intercept) & 7.77778 & 0.05435 & 143.099 & $<0.000^{* * *}$ \\
pca1PC1 & 0.03851 & 0.01377 & 2.796 & $0.00532^{* *}$ \\
pca1PC2 & -0.23416 & 0.01866 & -12.551 & $<0.000^{* * *}$ \\
pca1PC3 & 0.76260 & 0.02638 & 28.908 & $<0.000^{* * *}$ \\
pca1PC4 & 0.36481 & 0.02806 & 13.002 & $<0.000^{* * *}$ \\
pca1PC5 & 0.22498 & 0.03547 & 6.342 & $<0.000^{* * *}$ \\
pca1PC6 & -0.40160 & 0.03793 & -10.588 & $<0.000^{* * *}$ \\
pca1PC7 & 0.40160 & 0.03793 & 10.588 & $<0.000^{* * *}$ \\
pca1PC8 & 0.33451 & 0.04307 & 7.767 & $<0.000^{* * *}$ \\
\hline
\end{tabular}

Residual standard error: 1.422 on 675 degrees of freedom; Multiple R-squared: 0.685; Adjusted R-squared: 0.6813; F-statistic: 183.5 on 8 and 675 DF, $p$-value: $<0.000$. Signif. codes: ${ }^{* *}<0.000,{ }^{* *}<0.001$. 
The model conformed to the observed data (adjusted R-squared: 0.6813), and its main components were significant, $p$-value: $<0.000$. The multiple linear regression model would be

$\xi($ EFFECTIVENESS $)=7.777+0.038 \mathrm{CP} 1-0.23416 \mathrm{CP} 2+0.76260 \mathrm{CP} 3+0.36481 \mathrm{CP} 4+0.22498 \mathrm{CP} 5-$

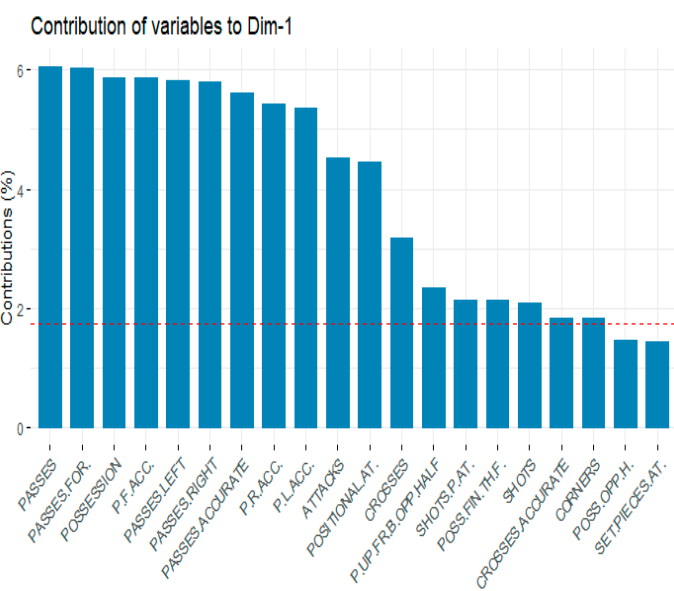

(a). Component 1: Passes

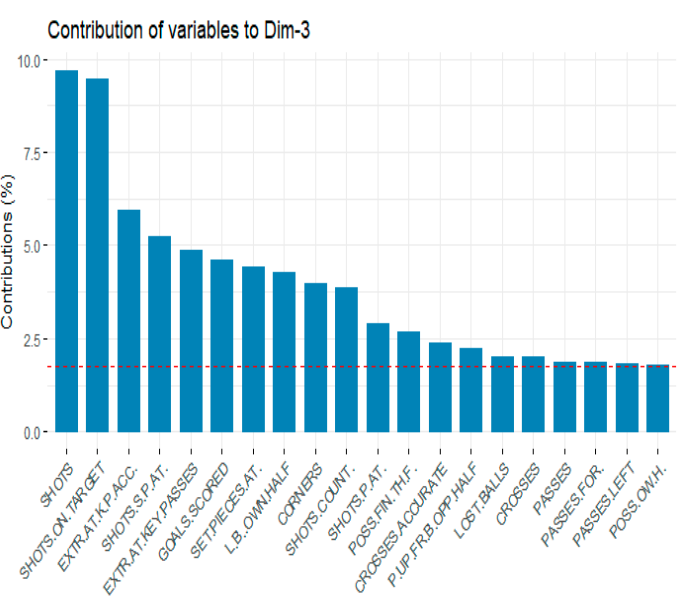

(c). Component 3: Shots

Contribution of variables to Dim-6

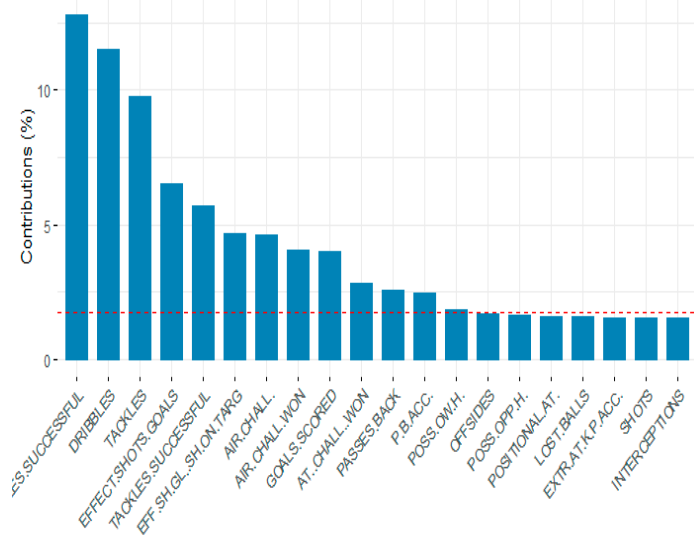

(e). Component 5: 1vs1

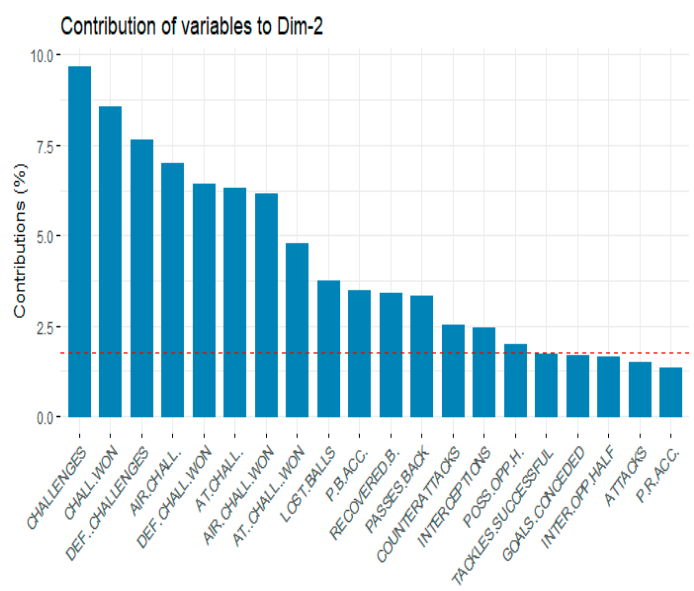

(b). Component 2: Challenges

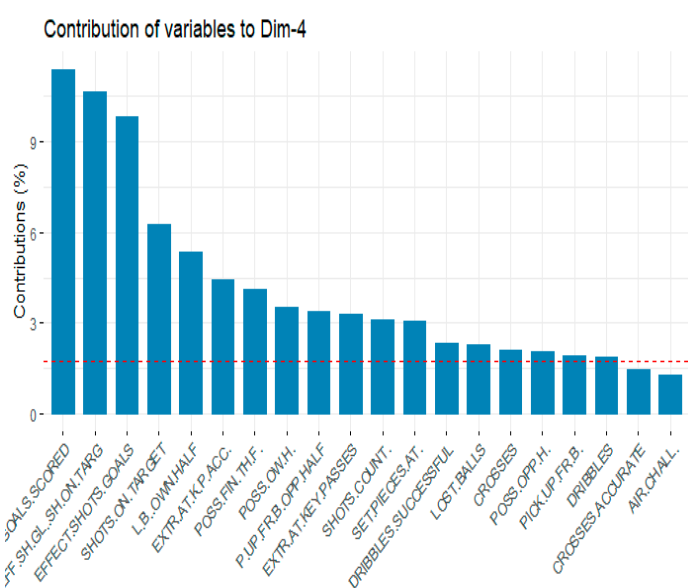

(d). Component 4: Attack effectiveness Contribution of variables to Dim-5

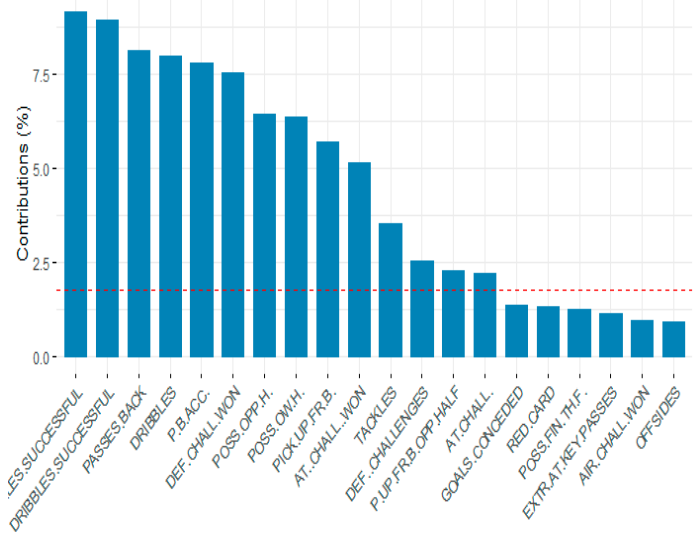

(f). Component 6: Dribbles

Figure 4. Cont. 


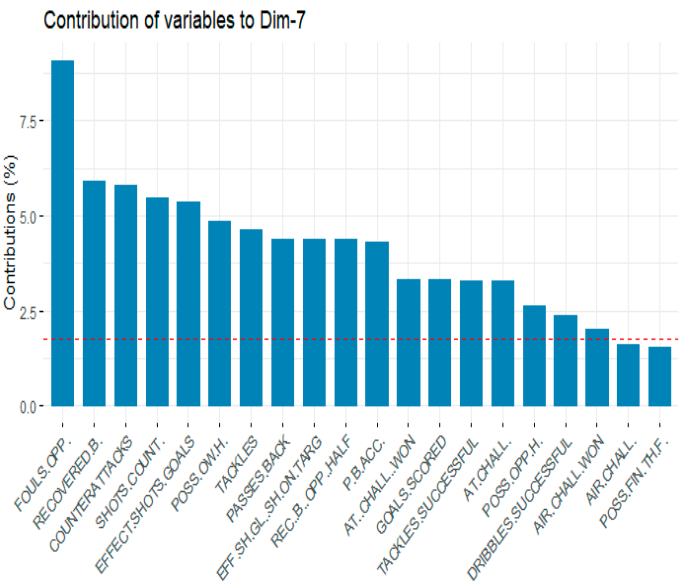

(g). Component 7: Fouls

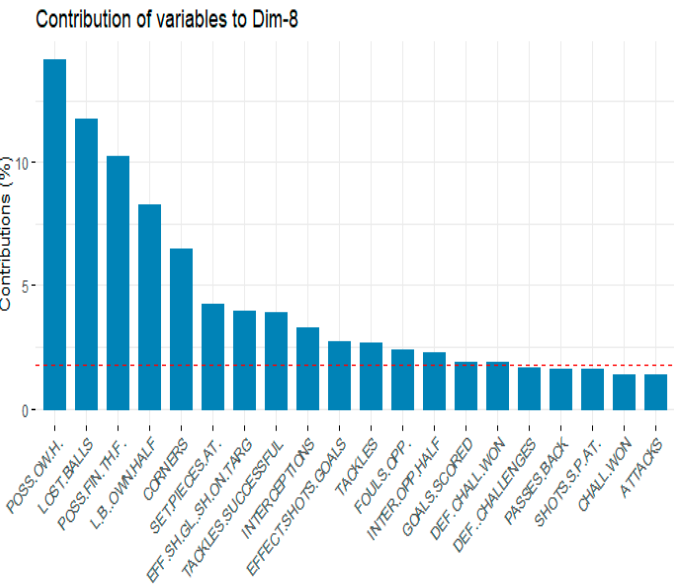

(h). Component 8: Possession

Contribution of variables to Dim-9

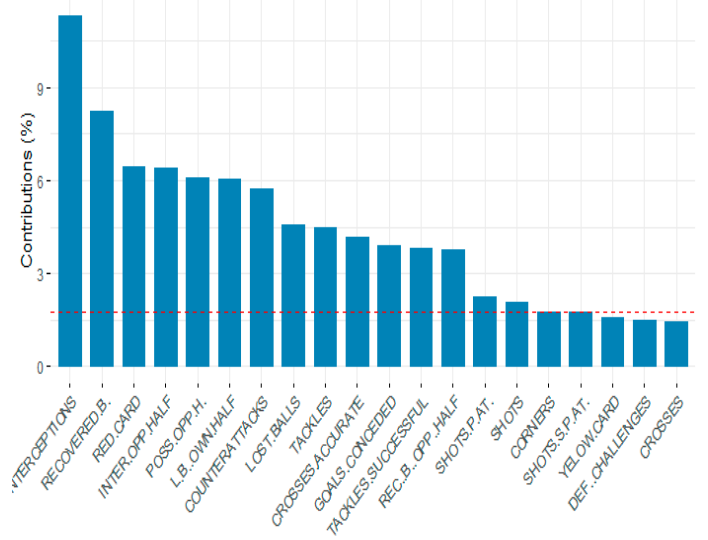

(i). Component 9: Offensive transitions

Figure 4. Components and new subjective variables of bottom teams. (a): Passes; (b): Challenges; (c): Shots; (d): Attacks effectiveness; (e): 1 vs 1; (f): Dribbles; (g): Fouls; (h): Possession balls; (i): Offensive transitions.

\subsection{Bottom Teams ANOVA and Linear Regression Model}

In Table 8 we can see how significant differences have been identified among the nine main components of the bottom teams.

Table 8. ANOVA bottom teams.

\begin{tabular}{cccccc}
\hline Model & Sum of Squares & Df & Mean Square & F & Sig. \\
\hline Regression & 86,366 & 9 & 9596 & 357,605 & $0.000^{\mathrm{b}}$ \\
Residual & 37,434 & 1395 & 0.027 & & \\
Total & 123,800 & 1404 & & & \\
\hline
\end{tabular}

Dependent Variable: EFFECTIVENESS; ${ }^{\text {. }}$ Predictors: (Constant), BottomPC1, BottomPC2, BottomPC3, BottomPC4, BottomPC5, BottomPC6, BottomPC7, BottomPC8, BottomPC9.

Residuals must be distributed randomly around 0 . The Shapiro-Wilk test affirmed the normality of the residuals ( $p$-value $=0.262$ ). The Breusch-Pagan test values were $\mathrm{BP}=1.8871, \mathrm{df}=2$, and a $p$-value $=0.389$, from which it follows that there was no evidence of a lack of homoscedasticity. The statistic values $\mathrm{D}-\mathrm{W}=1989, p$-value 0.972 , showed no evidence of autocorrelation.

Once the conditions in the residuals were checked, the linear regression model in unsuccessful teams was established (Table 9). 
Table 9. Bottom teams linear regression model.

\begin{tabular}{ccccc}
\hline Components & Estimate Std & Error & $\boldsymbol{t}$ Value & $\operatorname{Pr}(>|\mathbf{t}| \mathbf{)}$ \\
\hline (Intercept) & 6.72715 & 0.04504 & 149.352 & $<0.000^{* * *}$ \\
pca1PC1 & 0.17582 & 0.01232 & 14.273 & $<0.000^{* * *}$ \\
pca1PC2 & 0.07051 & 0.01575 & 4.478 & $<0.000^{* * *}$ \\
pca1PC3 & 0.30873 & 0.02157 & 14.312 & $<0.000^{* * *}$ \\
pca1PC4 & -0.84295 & 0.02448 & -34.433 & $<0.000^{* * *}$ \\
pca1PC5 & -0.18340 & 0.02786 & -6.582 & $<0.000^{* * *}$ \\
pca1PC6 & 0.55955 & 0.02951 & 18.964 & $<0.000^{* * *}$ \\
pca1PC7 & -0.54061 & 0.03054 & -17.704 & $<0.000^{* * *}$ \\
pca1PC8 & 0.51367 & 0.03279 & 15.665 & $<0.000^{* * *}$ \\
pca1PC9 & 0.05140 & 0.03587 & 1.433 & 0.152 \\
\hline Signif. codes: ${ }^{* * *}<0.000$. & & & &
\end{tabular}

The model conformed to the observed data (adjusted R-squared: 0.6813), and its main components were significant, $p$-value: $<0.000$. The multiple linear regression model would be

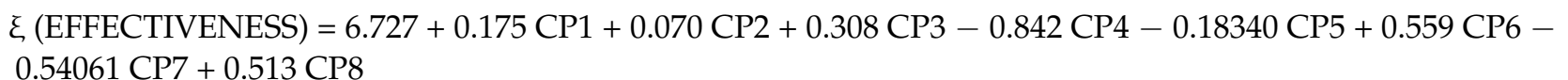

\section{Discussion}

To identify the indicators that influence football performance we perform a comparative analysis between teams of different levels of success, but sometimes we find a set of data with many related categories; therefore, the application of techniques that reduce the quantity of data could be useful. In this work we have considered reducing the dimensions of a data matrix without the loss of relevant information, using PCA. Subsequently we have used these PCs to try to identify the difference in performance between the best and bottom teams of LaLiga.

The PCA data mining technique allowed reducing the dimensions of a broad set of original categories without losing information, creating new categories for both groups. Specifically, we managed to reduce the original data matrix, composed of 57 categories in less than 10 new categories and with an explanation of the variance of $\geq 70 \%$, enabling the grouping of information and the simplification of the analysis. For the best teams group, PCA created 8 PCs that explained $70.1 \%$ of the variance (Table 1$)$ : Passes $(0.27 \%)$; Challenges (0.15\%); Attack effectiveness ( $0.08 \%)$; Shots $(0.07 \%)$; Dribbles $(0.04 \%)$; Tackles $(0.04 \%)$; Offensive transitions $(0.03 \%)$ and Possession $(0.03 \%)$. For the bottom teams group, 9 PCs were created that explained $70 \%$ of the variance (Table 3): Passes (0.23); Challenges $(0.14 \%)$; Shots (0.08\%); Attacks effectiveness (0.06\%); 1vs1 (0.05\%); Dribbles (0.04\%); Fouls opponent $(0.04 \%)$; Possession $(0.03 \%)$ and Offensive transitions $(0.03 \%)$.

In both groups, the Passes PC is denominated this way because most of the categories that constitute it refer to the number of passes and the time of possession. Challenges PC received this name because it included all types of challenges. The Attack effectiveness PC collected categories of the offensive phase, especially related to goals, shots and the effectiveness of shots. The Shots PC mainly included categories related to goals, shots, possession and passes. The Dribbles PC was mainly constituted by categories referring to dribbling, tackles and challenges. The Tackles PC was related to dribbling, challenges, tackles and lost balls. The Offensive transitions PC received this name for being related to recoveries, interceptions and counterattacks. The Possession PC, in the group of successful teams, is the one that showed a worse definition since it is made up of categories with less relation between them. In the bottom teams the 1vs1 PC included all dribbles and tackles. Fouls opponent PC is constituted by varied categories, being the heaviest ones the fouls opponent and, finally, Possession PC is also formed by different categories, the time of possession being the most important. 
Therefore, the PCA was shown, as in some previous works [23,31,33-35,45-47], as a good statistical technique, when we intend to reduce large data sets that have many interrelated variables, allowing us not only to speak of individual performance indicators, but of a set of related indicators.

If we use the PCs to compare the game of both groups, the first difference we observe is that, to explain the same percentage of variance, for the best teams group we need eight PCs, and for the bottom teams we need nine PCs. In both groups, both the category constituted from PC and called Passes, as well as Challenges, were those that allowed explaining the highest percentage of the variance. The Passes category had a slightly greater weight $(27 \%)$ in the best teams group than in the bottom teams (23\%) (Tables 2 and 4). On the other hand, Challenges showed a similar weight in both groups (15\% and $14 \%)$. However, the loadings of each PC were not exactly the same for each group (Tables 3 and 5). Thus, for Passes PC in the best teams group, the most important categories were passes, passes accurate, passes accurate left and passes accurate right. For the bottom teams, the highest weight categories were possession, passes, passes forward, passes left, passes right and passes forward accurate. Therefore, we can indicate that successful teams are characterized more by the efficiency of the passes than by the number of passes executed. That is, they have a greater number of successful passes than lower level teams. These results coincide with some previous works [22], but they analyzed 2014 Brazil FIFA World Cup and used a logistic regression. For Challenges PC we have also found some differences. It can be seen how, for the best teams, the attack challenges had greater weight; however, the defensive challenges were the ones most relevant for bottom teams. This circumstance can be explained because the bottom teams are characterized by staying longer in the defensive phase, executing many more defensive than offensive actions. Previous work also coincides in indicating that the successful teams show higher averages of offensive variables, and unsuccessful teams show higher averages of defensive variables [48].

Another difference that we can see in terms of PC formation is that in the best teams the PC called Tackles is formed, consisting mainly of the categories dribbling, challenges, tackles and lost balls. In the bottom teams the 1vs1 PC and fouls were constituted but did not appear in the other group. In spite of these differences we can appreciate that both the components constituted for both groups, as well as the categories and the weight of these in each component, were very similar. This circumstance leads us to think that in high level football the differences between the teams are minimal, and their success or failure may be explained by the individual performance of their players.

The results of the linear regression model (Tables 5 and 7) allow us to identify which PCs have the greatest influence on the performance of both groups of teams. For this, a prediction model of the category "EFFECTIVENESS" was built, both for the best and for the bottom teams. The linear regression model of the best teams group, ordering the PCs from highest to lowest weight, was constituted as follows: Attack effectiveness (0.76260); Offensive transitions (0.40160); Shots (0.36481); Possession (0.33451); Dribbles (0.22498); Passes (0.03851); Challenges $(-0.23416)$ and Tackles $(-0.40160)$. In the bottom teams the order was as follows: Dribbles (0.55955); Possession (0.51367); Shots (0.30873); Passes (0.17582); Challenges (0.07051); 1vs1 (-0.18340); Fouls ( -0.54061$)$ and Attack effectiveness $(-0.84295)$. We can see how in best teams, the PC that offered a greater influence on the prediction of this category was Attack effectiveness. The number of goals, a greater ball possession time in the final third of the field, a greater number of effective shots and crosses allow to increase the performance in best teams. This information is essential for technicians since, if they manage to improve the performance of their teams in these elements of the game, they will increase their offensive performance. The information provided by the number of goals is trivial since it is obvious that scoring more goals implies increasing offensive performance, but the other indicators referring to ball possession zone, effective shots and crosses do offer transcendent information. These results are corroborated by the works of $[9,10]$ who indicated that successful teams have longer-term possessions in the middle of the offensive field than the defensive one. The works $[19,22,49,50]$ indicate 
that successful teams show greater effectiveness in shooting, also ratify in their work that making a greater number of crosses increases the chances of winning the matches. In contrast to the cited studies, in our work we have obtained similar results using a different method, specifically through a data mining technique. Winter and Pfeiffer [23] also reached the same conclusion in their work, indicating that there is a relationship between offense efficiency and success, but they analyzed UEFA Euro 2012 and considered success as the match outcome.

Following the results of the linear regression model, we can indicate how the main differences in the prediction of performance of both groups occur in PCs offensive transitions, tackles, challenges, dribbles, fouls opponent and 1vs1. Offensive transitions play a more important role in the best teams than in the bottom teams. Thus, in the best teams, performing a greater number of recoveries, interceptions and counterattacks, that is, dynamic offensive transitions through counterattacks, would increase their performance in the game. This circumstance was also pointed out by Tenga et al. [40]. These authors analyzed the Norwegian league, and by means of a multiple linear regression, they obtained that the proportion of goals scored during counterattacks (52\%) was higher than during elaborate attacks (48\%). Therefore, the offensive game seems to be more efficient against a disorderly defense. This information is very important for the coaches, who should focus their training on these game situations, both in attack and defense, to try to improve their performance in both phases of the game.

In the best teams the Tackles and Challenges PCs negatively influenced the offensive performance. This may be due to the fact that these are more typical behaviors of unsuccessful teams, as indicated above [48].

In bottom teams it was appreciated how increasing the number of successful dribblings would increase performance. This result coincides with that of the work of Harrop and Nevill [21] who found that the number of dribbles is correlated with performance. The PC Fouls opponent also showed a strong negative influence on the performance of bottom teams and that these teams showed fewer effective attacks than the best teams.

We have achieved the aims set and the sample used, as these are the matches of three competitive seasons, allowing us to generalize the results. The main contribution and novelty of this work is that we have carried out a longitudinal tactical analysis of LaLiga teams, using the combination of factor analysis and linear regression. However, we believe that the differences found in the constitution of the different PCs have not been as satisfactory as we would have liked. We believe that this may be due to the design used, in our case we have found the PCs for each group of teams separately and, subsequently, we have tried to build a probabilistic model with the detected PCs. In future works we should propose a design in which we find the main components for both groups and then build a separate model for each group. In addition, since the goals scored and received did not have a significant contribution to the main components, in the future it could be considered to eliminate these variables from the analysis because this approach may be biasing the same.

The results of this work offer information to the technicians, about what are the KPIs in football and the game pattern of the best teams, being able to compare the latter with that of their own teams, and thus, to be able to make the appropriate modifications, to increase performance.

\section{Conclusions}

The realization of this work has allowed us, with the use of the PCA, to reduce a dimension of data without losing relevant information. We have been able to identify the KPI of the best and bottom teams, and we have identified the main differences between both groups.

Best teams are characterized and differentiated from bottom teams in the realization of a greater number of successful passes and in the execution of a greater number of dynamic offensive transitions. 
Bottom teams are characterized by executing more defensive than offensive actions.

A greater ball possession time in the final third of the field, and a greater number of effective shots and crosses, are the main performance factors that influence the offensive success of football.

Author Contributions: Conceptualization, C.A.C. and J.L.L.; methodology, C.A.C. and J.L.L.; formal analysis, J.L.L. and D.B.; investigation, C.A.C. and J.L.L.; writing-original draft preparation, C.A.C. and J.L.L.; writing-review and editing, C.A.C., J.L.L., D.B. and R.M.; supervision, D.B. and R.M.; project administration, C.A.C. All authors have read and agreed to the published version of the manuscript.

Funding: This research received no external funding.

Institutional Review Board Statement: Not applicable.

Informed Consent Statement: Not applicable.

Data Availability Statement: Data was obtained from InStatscout and are available from www. instatscout.com (accessed on 19 March 2021).

Conflicts of Interest: The authors declare no conflict of interest.

\section{References}

1. Hughes, M.; Bartlett, R. What is performance analysis? In The Essentials of Performance Analysis: An Introduction; Hughes, M., Franks, I., Eds.; Routledge: London, UK, 2008; pp. 8-20.

2. Hughes, M.D.; Bartlett, R.M. The use of performance indicators in performance analysis. J. Sports Sci. 2002, 20, 739-754. [CrossRef]

3. Carling, C.; Wright, C.; Nelson, L.J.; Bradley, P. Comment on 'Performance analysis in football: A critical review and implications for future research'. J. Sports Sci. 2014, 32, 2-7. [CrossRef]

4. O'Donoghue, P. Normative profiles of sports performance. Int. J. Perform. Anal. Sport 2005, 5, 104-119. [CrossRef]

5. Carling, C.; Reilly, T.; Williams, A.M. Performance Assessment for Field Sports; Routledge: London, UK, 2009.

6. O'Donoghue, P. Sports performance profiling. In Routledge Handbook of Sport Performance Analysis; McGarry, T., O'Donoghue, P., Sampaio, J., Eds.; Routledge: London, UK, 2013; pp. 127-139.

7. Vilar, L.; Araújo, D.; Davids, K.; Button, C. The Role of Ecological Dynamics in Analysis Performance in Teams Sports. Sports Med. 2012, 42, 1-10. [CrossRef] [PubMed]

8. Mackenzie, R.; Cushion, C. Performance analysis in football: A critical review and implications for future research. J. Sports Sci. 2013, 31, 639-676. [CrossRef] [PubMed]

9. Casal, C.A.; Anguera, M.T.; Maneiro, R.; Losada, J.L. Possession in Football: More Than a Quantitative Aspect-A Mixed Method Study. Front. Psychol. 2019, 10, 201. [CrossRef] [PubMed]

10. Casal, C.A.; Maneiro, R.; Ardá, T.; Marí, F.J.; Losada, J.L. Possession Zone as a Performance Indicator in Football. The Game of the Best Teams. Front. Psychol. 2017, 8, 1176. [CrossRef] [PubMed]

11. Castellano, J. Relación entre indicadores de rendimiento y éxito en el fútbol profesional. Rev. Iberoam. Psicol. Ejerc. Deporte 2018, $13,41-49$.

12. Hook, C.; Hughes, M. Patterns of Play Leading to Shots in 'Euro 2000'; Passcom UWIC: Cardiff, UK, 2001.

13. Horn, R.; Williams, M.; Ensum, J. Attacking in central areas: A preliminary analysis of attacking play in the 2001/2002 FA Premiership season. Insight 2002, 3, 31-34.

14. Hughes, M.D.; Churchill, S. Attacking profiles of successful and unsuccessful teams in Copa America 2001. In Science and Football $V$; Reilly, T., Cabri, J., Araujo, D., Eds.; Routledge: Abingdon, UK, 2005; pp. 219-224.

15. Jones, P.D.; James, N.; Mellalieu, S.D. Possession as a performance indicator in soccer. Int. J. Perform. Anal. Sport 2004, 4, 98-102. [CrossRef]

16. Lago-Peñas, C.; Lago-Ballesteros, J.; Rey, E. Differences in performance indicators between winning and losing teams in the UEFA Champions League. J. Hum. Kinet. 2011, 27, 135-146. [CrossRef]

17. Stanhope, J. An investigation into possession with respect to time in the Soccer World Cup 1994. In Notational Analysis of Sport III; Hughes, M.D., Ed.; UWIC: Cardiff, UK, 2001; pp. 155-162.

18. Bekris, E.; Gioldasis, A.; Gissis, I.; Komsis, S.; Alipasali, F. Winners and losers in top level soccer. How do they differ? J. Phys. Educ. Sport 2014, 14, 398-405.

19. Castellano, J.; Casamichana, D.; Lago, C. The Use of Match Statistics that Discriminate Between Successful and Unsuccessful Soccer Teams. J. Hum. Kinet. 2012, 31, 137-147. [CrossRef]

20. Collet, C. The possession game? A comparative analysis of ball retention and team success in European and international football, 2007-2010. J. Sports Sci. 2013, 31, 123-136. [CrossRef] [PubMed]

21. Harrop, K.; Nevill, A. Performance indicators that predict success in an English professional League One soccer team. Int. J. Perform. Anal. Sport 2014, 14, 907-920. [CrossRef] 
22. Liu, H.; Gomez, M.-Á.; Lago-Peñas, C.; Sampaio, J. Match statistics related to winning in the group stage of 2014 Brazil FIFA World Cup. J. Sports Sci. 2015, 33, 1205-1213. [CrossRef]

23. Winter, C.; Pfeiffer, M. Tactical metrics that discriminate winning, drawing and losing teams in UEFA Euro $2012{ }^{\circledR}$. J. Sports Sci. 2016, 34, 486-492. [CrossRef]

24. Adams, D.; Morgans, R.; Sacramento, J.; Morgan, S.; Williams, M.D. Successful short passing frequency of defenders differentiates between top and bottom four English Premier League teams. Int. J. Perform. Anal. Sport 2013, 13, 653-668. [CrossRef]

25. Armatas, V.; Yiannakos, A.; Zaggelidis, G.; Skoufas, D.; Papadopoulou, S.; Fragkos, N. Differences in offensive actions betwen top and last teams in Greek first soccer division. A retrospective study 1998-2008. J. Phys. Educ. Sport 2009, 23, 1-5.

26. Bradley, P.S.; Archer, D.T.; Hogg, B.; Schuth, G.; Bush, M.; Carling, C.; Barnes, C. Tier-specific evolution of match performance characteristics in the English Premier League: It's getting tougher at the top. J. Sports Sci. 2016, 34, 980-987. [CrossRef]

27. Hoppe, M.; Slomka, M.; Baumgart, C.; Weber, H.; Freiwald, J. Match Running Performance and Success across a Season in German Bundesliga Soccer Teams. Int. J. Sports Med. 2015, 36, 563-566. [CrossRef] [PubMed]

28. Lago-Ballesteros, J.; Lago, C. Performance in team sports: Identifying the keys to success in soccer. J. Hum. Kinet. 2010, 25, 85-95. [CrossRef]

29. Lozares Colina, C.; López Roldán, P. El análisis de componentes principales: Aplicación al análisis de datos secundarios. Pap. Rev. Sociol. 1991, 37, 31. [CrossRef]

30. Gómez, M.A.; Gómez-Lopez, M.; Lago, C.; Sampaio, J. Effects of game location and final outcome on game-related statistics in each zone of the pitch in professional football. Eur. J. Sport Sci. 2012, 12, 393-398. [CrossRef]

31. Moura, F.A.; Martins, L.E.B.; Cunha, S.A. Analysis of football game-related statistics using multivariate techniques. J. Sports Sci. 2014, 32, 1881-1887. [CrossRef] [PubMed]

32. Carpita, M.; Sandri, M.; Simonetto, A.; Zuccolotto, P. Discovering the Drivers of Football Match Outcomes with Data Mining. Qual. Technol. Quant. Manag. 2015, 12, 561-577. [CrossRef]

33. Ric, A.; Torrents, C.; Gonçalves, B.; Sampaio, J.E.; Hristoski, R. Soft-assembled Multilevel Dynamics of Tactical Behaviors in Soccer. Front Psychol. 2016, 7, 1513. [CrossRef] [PubMed]

34. Fernandez-Crehuet, J.M.; Rosales-Salas, J.; Navarro, P. A performance index of football teams: The Spanish case. Sport Soc. 2019, 1-23. [CrossRef]

35. Lago-Peñas, C.; Gómez-Ruano, M.; Yang, G. Styles of play in professional soccer: An approach of the Chinese Soccer Super League. Int. J. Perform. Anal. Sport 2017, 17, 1073-1084. [CrossRef]

36. The Belmont Report. Ethical Principles and Guidelines for the Protection of Human Subjects of Research. Available online: https: / / www.hhs.gov/ohrp/regulations-and-policy/belmont-report/index.html (accessed on 19 March 2021).

37. Liu, H.; Hopkins, W.; Gómez, M.A.; Molinuevo, J.S. Inter-operator reliability of live football match statistics from OPTA Sportsdata. Int. J. Perform. Anal. Sport 2013, 13, 803-821. [CrossRef]

38. Tenga, A.; Holme, I.; Ronglan, L.T.; Bahr, R. Effect of playing tactics on goal scoring in Norwegian professional soccer. J. Sports Sci. 2010, 28, 237-244. [CrossRef]

39. Tenga, A.; Holme, I.; Ronglan, L.T.; Bahr, R. Effect of playing tactics on achieving score-box possessions in a random series of team possessions from Norwegian professional soccer matches. J. Sports Sci. 2010, 28, 245-255. [CrossRef]

40. Tenga, A.; Ronglan, L.T.; Bahr, R. Measuring the effectiveness of offensive match-play in professional soccer. Eur. J. Sport Sci. 2010, 10, 269-277. [CrossRef]

41. Kaiser, H.F. The varimax criterion for analytic rotation in factor analysis. Psychometrika 1958, 23, 187-200. [CrossRef]

42. Weaving, D.; Jones, B.; Marshall, P.; Till, K.; Abt, G. Multiple Measures are Needed to Quantify Training Loads in Professional Rugby League. Int. J. Sports Med. 2017, 38, 735-740. [CrossRef]

43. Coutts, A.J. Working Fast and Working Slow: The Benefits of Embedding Research in High-Performance Sport. Int. J. Sports Physiol. Perform. 2016, 11, 1-2. [CrossRef] [PubMed]

44. Venables, W.N.; Ripley, B.D. Modern Applied Statistics with S, 4th ed.; Springer: New York, NY, USA, 2002.

45. Higueras-Herbada, A.; Travieso, D.; Ibáñez-Gijón, J.; Jacobs, D.M. Anticipating the Lateral Direction of Penalty Kicks in Football From PCA-Reduced Point-Light Displays. Ecol. Psychol. 2017, 29, 23-34. [CrossRef]

46. Nakamura, T.; Miyoshi, T.; Sato, S.; Takagi, M.; Kamada, Y.; Kobayashi, Y. Differences in soccer kicking type identified using principal component analysis. Sports Eng. 2018, 21, 149-159. [CrossRef]

47. Lago-Peñas, C.; Lago-Ballesteros, J.; Dellal, A.; Gómez, M. Game-related statistics that discriminated winning, drawing and losing teams from the Spanish soccer league. J. Syst. Sci. Med. 2010, 9, 288-293.

48. Delgado-Bordonau, J.L.; Domenech-Monforte, C.; Guzman, J.F.; Mendez-Villanueva, A. Offensive and defensive team performance: Relation to successful and unsuccessful participation in the 2010 Soccer World Cup. J. Hum. Sport Exerc. 2013, 8, 894-904. [CrossRef]

49. Szwarc, A. Effectiveness of Brazilian and German teams and the teams defeated by them during the 17th FIFA World Cup. Kinesiology 2004, 36, 83-89.

50. Yue, Z.; Broich, H.; Mester, J. Statistical Analysis for the Soccer Matches of the First Bundesliga. Int. J. Sports Sci. Coach. 2014, 9 , 553-560. [CrossRef] 